Asset Prices in an Exchange Economy

Author(s): Robert E. Lucas, Jr.

Source: Econometrica, Vol. 46, No. 6 (Nov., 1978), pp. 1429-1445

Published by: The Econometric Society

Stable URL: http://www.jstor.org/stable/1913837

Accessed: $1 2 \longdiv { / 0 3 / 2 0 1 0 0 5 : 0 9 }$

Your use of the JSTOR archive indicates your acceptance of JSTOR's Terms and Conditions of Use, available at http://www.jstor.org/page/info/about/policies/terms.jsp. JSTOR's Terms and Conditions of Use provides, in part, that unless you have obtained prior permission, you may not download an entire issue of a journal or multiple copies of articles, and you may use content in the JSTOR archive only for your personal, non-commercial use.

Please contact the publisher regarding any further use of this work. Publisher contact information may be obtained at http://www.jstor.org/action/showPublisher?publisherCode=econosoc.

Each copy of any part of a JSTOR transmission must contain the same copyright notice that appears on the screen or printed page of such transmission.

JSTOR is a not-for-profit service that helps scholars, researchers, and students discover, use, and build upon a wide range of content in a trusted digital archive. We use information technology and tools to increase productivity and facilitate new forms of scholarship. For more information about JSTOR, please contact support@jstor.org. 


\title{
ASSET PRICES IN AN EXCHANGE ECONOMY
}

\author{
By ROBERT E. LUCAS, JR. ${ }^{1}$
}

\begin{abstract}
This paper is a theoretical examination of the stochastic behavior of equilibrium asset prices in a one-good, pure exchange economy with identical consumers. A general method of constructing equilibrium prices is developed and applied to a series of examples.
\end{abstract}

\section{INTRODUCTION}

THIS PAPER IS A THEORETICAL examination of the stochastic behavior of equilibrium asset prices in a one-good, pure exchange economy with identical consumers. The single good in this economy is (costlessly) produced in a number of different productive units; an asset is a claim to all or part of the output of one of these units. Productivity in each unit fluctuates stochastically through time, so that equilibrium asset prices will fluctuate as well. Our objective will be to understand the relationship between these exogenously determined productivity changes and market determined movements in asset prices.

Most of our attention will be focused on the derivation and application of a functional equation in the vector of equilibrium asset prices, which is solved for price as a function of the physical state of the economy. This equation is a generalization of the Martingale property of stochastic price sequences, which serves in practice as the defining characteristic of market "efficiency," as that term is used by Fama [7] and others. The model thus serves as a simple context for examining the conditions under which a price series' failure to possess the Martingale property can be viewed as evidence of non-competitive or "irrational" behavior.

The analysis is conducted under the assumption that, in Fama's terms, prices "fully reflect all available information," an hypothesis which Muth [13] had earlier termed "rationality of expectations." As Muth made clear, this hypothesis (like utility maximization) is not "behavioral": it does not describe the way agents think about their environment, how they learn, process information, and so forth. It is rather a property likely to be (approximately) possessed by the outcome of this unspecified process of learning and adapting. One would feel more comfortable, then, with rational expectations equilibria if these equilibria were accompanied by some form of "stability theory" which illuminated the forces which move an economy toward equilibrium. The present paper also offers a convenient context for discussing this issue.

The conclusions of this paper with respect to the Martingale property precisely replicate those reached earlier by LeRoy (in [10] and [11]), and not surprisingly, since the economic reasoning in $[\mathbf{1 0}]$ and the present paper is the same. The

\footnotetext{
${ }^{1}$ This paper originated in a conversation with Pentti Kouri, who posed to me the problem studied below. I would also like to thank Yehuda Freidenberg, Jose Scheinkman, and Joseph Williams for many helpful comments.
} 
context used here differs somewhat from LeRoy's, however, and the analytical methods used differ considerably.

The economy is informally described in the next section, and equilibrium is formally defined in Section 3. In Section 4, the basic functional equation for prices is derived and studied. Section 5 develops a certain "duality" property, on which is based the discussion of stability in Section 6. Section 7 deals with examples which are simple enough to permit either explicit solution or some "comparative static" exercises. The role of the Martingale property is discussed in Section 8. Section 9 concludes the paper.

\section{DESCRIPTION OF THE ECONOMY}

Consider an economy with a single consumer, interpreted as a representative "stand in" for a large number of identical consumers. He wishes to maximize the quantity

$$
E\left\{\sum_{t=0}^{\infty} \beta^{t} U\left(c_{t}\right)\right\}
$$

where $c_{t}$ is a stochastic process representing consumption of a single good, $\beta$ is a discount factor, $U(\cdot)$ is a current period utility function, and $E\{\cdot\}$ is an expectations operator.

The consumption good is produced on $n$ distinct productive units. Let $y_{i t}$ be the output of unit $i$ in period $t, i=1, \ldots, n$, and let $y_{t}=\left(y_{1 t}, \ldots, y_{n t}\right)$ be the output vector in $t$. Output is perishable, so that feasible consumption levels are those which satisfy

$$
0 \leqslant c_{t} \leqslant \sum_{i=1}^{n} y_{i t} .
$$

Production is entirely "exogenous": no resources are utilized, and there is no possibility of affecting the output of any unit at any time. The motion of $y_{t}$ will be taken to follow a Markov process, defined by its transition function

$$
F\left(y^{\prime}, y\right)=\operatorname{pr}\left\{y_{t+1} \leqslant y^{\prime} \mid y_{t}=y\right\} .
$$

Ownership in these productive units is determined each period in a competitive stock market. Each unit has outstanding one perfectly divisible equity share. A share entitles its owner as of the beginning of $t$ to all of the unit's output in period $t$. Shares are traded, after payment of real dividends, at a competitively determined price vector $p_{t}=\left(p_{1 t}, \ldots, p_{n t}\right)$. Let $z_{t}=\left(z_{1 t}, \ldots, z_{n t}\right)$ denote a consumer's beginning-of-period share holdings.

In this economy, it is easy to determine equilibrium quantities of consumption and asset holdings. All output will be consumed $\left(c_{t}=\Sigma_{i} y_{i t}\right)$ and all shares will be held $\left(z_{t}=(1,1, \ldots, 1)=1\right.$ for all $\left.t\right)$. The main analytical issue, then, will be the determination of equilibrium price behavior.

Our attack on this problem begins from the observation that all relevant information on the current and future physical state of the economy is sum- 
marized in the current output vector $y$. Since, given recursive preferences, the asset market "solves" a problem of the same form each period, equilibrium prices should (if they behave in a systematic way at all) be expressible as some fixed function $p(\cdot)$ of the state of the economy, or $p_{t}=p\left(y_{t}\right)$ where the $i$ th coordinate $p_{i}\left(y_{t}\right)$ is the price of a share of unit $i$ when the economy is in the state $y_{t}$. If so, knowledge of the transition function $F\left(y^{\prime}, y\right)$ and this function $p(y)$ will suffice to determine the stochastic character of the price process $\left\{p_{t}\right\}$.

Similarly, one would expect a consumer's current consumption and portfolio decisions, $c_{t}$ and $z_{t+1}$, to depend on his beginning of period portfolio, $z_{t}$, the prices he faces, $p_{t}$, and the relevant information he possesses on current and future states of the economy, $y_{t}$. If so, his behavior can be described by fixed decision rules $c(\cdot)$ and $g(\cdot): c_{t}=c\left(z_{t}, y_{t}, p_{t}\right)$ and $z_{t+1}=g\left(z_{t}, y_{t}, p_{t}\right)$.

Now given perceived, future price behavior $F\left(y^{\prime}, y\right)$ and $p(y)$, consumers will be able to determine these decision rules $c(\cdot)$ and $g(\cdot)$ optimally. In this sense, a price function $p$ determines consumer behavior. On the other hand, given decision rules $c(\cdot)$ and $g(\cdot)$, the current period market clearing conditions determine a price function $p(\cdot)$. In this sense, consumer behavior determines the equilibrium price function. We close the system with the assumption of rational expectations: the market clearing price function $p$ implied by consumer behavior is assumed to be the same as the price function $p$ on which consumer decisions are based.

\section{DEFINITION OF EQUILIBRIUM}

The economy described in the preceding section is specified by the functions $U$ and $F$ and the number $\beta$. Assume $0<\beta<1 . U: R^{+} \rightarrow R^{+}$is continuously differentiable, bounded, increasing, and strictly concave, with $U(0)=0 .{ }^{2}$ $F: E^{n+} \times E^{n+} \rightarrow R$ is continuous; $F(\cdot, y)$ is a distribution function for each fixed $y$, with $F(0, y)=0$. Assume that the process defined by $F$ has a stationary distribution $\phi(\cdot)$, the unique solution to

$$
\phi\left(y^{\prime}\right)=\int F\left(y^{\prime}, y\right) d \phi(y),
$$

and that for any continuous function $g(y)$,

$$
\int g\left(y^{\prime}\right) d F\left(y^{\prime}, y\right)
$$

is a continuous function of $y$.

An equilibrium will be a pair of functions: a price function $p(y)$, as discussed above, and an optimum value function $v(z, y)$. The value $v(z, y)$ will be interpreted as the value of the objective (1) for a consumer who begins in state $y$ with holdings $z$, and follows an optimum consumption-portfolio policy thereafter.

\footnotetext{
${ }^{2} R^{+}$is the set of nonnegative real numbers. $E^{n}$ is $n$-dimensional space. $E^{n+}$ is the subset of $E^{n}$ with all components nonnegative $\left(x \in E^{n}\right.$ and $\left.x \geqslant 0\right) . L^{n}$ is the set of continuous, bounded functions with domain $E^{n}$, and so on.
} 
Definition: An equilibrium is a continuous function $p(y): E^{n+} \rightarrow E^{n+}$ and a continuous, bounded function $v(z, y): E^{n+} \times E^{n+} \rightarrow R^{+}$such that

$$
v(z, y)=\max _{c, x}\left\{U(c)+\beta \int v\left(x, y^{\prime}\right) d F\left(y^{\prime}, y\right)\right\}
$$

subject to

$$
c+p(y) \cdot x \leqslant y \cdot z+p(y) \cdot z, \quad c \geqslant 0, \quad 0 \leqslant x \leqslant \bar{z},
$$

where $\bar{z}$ is a vector with components exceeding one;

$$
\text { for each } y, v(1, y) \text { is attained by } c=\sum_{i} y_{i} \text { and } x=\underline{1} \text {. }
$$

Condition (i) says that, given the behavior of prices, a consumer allocates his resources $y \cdot z+p(y) \cdot z$ optimally among current consumption $c$ and end-ofperiod share holdings $x .^{3}$ Condition (ii) requires that these consumption and portfolio decisions be market clearing. Since the market is always cleared, the consumer will never be observed except in the state $z=1$. On the other hand, the consumer has (though he always rejects it) the option to choose security holdings $x \neq \underline{1}$. To evaluate these options, he needs to know $v(z, y)$ for all $z{ }^{4}$

\section{CONSTRUCTION OF THE EQUILIBRIUM}

We begin by studying the consumer's maximum problem (i) for given price behavior $p(y)$. We have the following proposition.

Proposition 1: For each continuous price function $p(\cdot)$ there is a unique, bounded, continuous, nonnegative function $v(z, y ; p)$ satisfying $(i)$. For each $y$, $v(z, y ; p)$ is an increasing, concave function of $z$.

Proof: Define the operator $T_{p}$ on functions $v(z, y)$ such that (i) is equivalent to $T_{p} v=v$. The domain of $T_{p}$ is the nonnegative orthant $L^{2 n+}$ of the space $L^{2 n}$ of continuous, bounded functions $u: E^{n+} \times E^{n+} \rightarrow R$, normed by

$$
\|u\|=\sup _{z, y}|u(z, y)| .
$$

Since applying $T_{p}$ involves maximizing a continuous function over a compact set, $T_{p} u$ is well defined for any $u \in L^{2 n+}$. Since $U(c)$ is bounded, $T_{p} u$ is bounded, and by $\left[2\right.$, p. 116] $T_{p} u$ is continuous. Hence $T_{p}: L^{2 n+} \rightarrow L^{2 n+} . T_{p}$ is monotone $(u \geqslant v$ implies $\left.T_{p} u \geqslant T_{p} v\right)$ and for any constant $A, T_{p}(u+A)=T_{p} u+\beta A$. Then from [3, Theorem 5] $T_{p}$ is a contraction mapping. It follows that $T_{p} v=v$ has a unique solution $v$ in $L^{2 n+}$, as was to be shown. Further, $\lim _{n \rightarrow \infty} T_{p}^{n} u=v$ for any $u \in L^{2 n+}$.

\footnotetext{
${ }^{3}$ The bound $\bar{z}$ on $x$ is to assure that the maximization in (i) is always over a compact set, even if some components of $p(y)$ are zero.

${ }^{4}$ This is not a "new" concept of equilibrium. It is (though no proof is offered) a standard, Arrow-Debreu equilibrium where the commodity space is the space of all possible realizations of the process $\Sigma_{i} y_{i t}$. See [12] for a full development of this relationship in a closely related context.
} 
To prove that $v$ is increasing in $z$, observe that $T_{p} u$ is an increasing function of $z$ for any $u$. Since $v=T_{p} v$, this implies that $v$ is increasing in $z$.

To prove that $v$ is concave in $z$, we first show that if $u(z, y)$ is concave in $z$, so is $\left(T_{p} u\right)(z, y)$. Let $z_{0}, z_{1}$ be chosen, let $0 \leqslant \theta \leqslant 1$, and let $z^{\theta}=\theta z^{0}+(1-\theta) z^{1}$. Let $\left(c_{i}, x_{i}\right)$ attain $\left(T_{p} u\right)\left(z^{i}, y\right), i=0,1$. Now $\left(c^{\theta}, x^{\theta}\right)=\left(\theta c^{0}+(1-\theta) c^{1}, \theta x^{0}+(1-\theta) x^{1}\right)$ satisfies $c^{\theta}+p(y) \cdot x^{\theta} \leqslant y \cdot z^{\theta}+p(y) \cdot z^{\theta}$, so that

$$
\begin{aligned}
\left(T_{p} u\right)\left(z^{\theta}, y\right) & \geqslant U\left(c^{\theta}\right)+\beta \int u\left(x^{\theta}, y^{\prime}\right) d F\left(y^{\prime}, y\right) \\
& \geqslant \theta\left(T_{p} u\right)\left(z^{0}, y\right)+(1-\theta)\left(T_{p} u\right)\left(z^{1}, y\right)
\end{aligned}
$$

using the concavity of $U$ and $u$. Hence $\left(T_{p} u\right)(z, y)$ is concave in $z$. It follows by an induction that $T_{p}^{n} u$ is concave in $z$ for all $n=1,2, \ldots$ Then, since $\lim _{n \rightarrow \infty} T_{p}^{n} u=v, v$ is concave.

The derivatives of $v$ with respect to $z$ are described in the following proposition.

Proposition 2: If $v(z, y ; p)$ is attained at $(c, x)$ with $c>0$, then $v$ is differentiable with respect to $z$ at $(z, y)$ and

$$
\frac{\partial v(z, y ; p)}{\partial z_{i}}=U^{\prime}(c)\left[y_{i}+p_{i}(y)\right] \quad(i=1, \ldots, n) .
$$

Proof: Define $f: R^{+} \rightarrow R^{+}$by

$$
f(A)=\max _{c, x}\left\{U(c)+\beta \int v\left(x, y^{\prime}\right) d F\left(y^{\prime}, y\right)\right\}
$$

subject to

$$
c+p(y) \cdot x \leqslant A, \quad c, x \geqslant 0 .
$$

For each $A, f(A)$ is attained at $c(A), x(A)$ say, and since the maximand is strictly concave in $c, c(A)$ is unique and varies continuously with $A$ [2, p. 116]. If $c(A)>0$ and if $h$ is sufficiently small, $c(A)+h$ is feasible at "income" $A+h$, and $c(A+h)-h$ is feasible at income $A$. Thus

$$
\begin{aligned}
f(A+h) & \geqslant u(c(A)+h)+\beta \int v\left(x(A), y^{\prime}\right) d F\left(y^{\prime}, y\right) \\
& =u(c(A)+h)-u(c(A))+f(A)
\end{aligned}
$$

and

$$
\begin{aligned}
f(A) & \geqslant u(c(A+h)-h)+\beta \int v\left(x(A+h), y^{\prime}, y\right) \\
& =u(c(A+h)-h)-u(c(A+h))+f(A+h) .
\end{aligned}
$$

Combining these inequalities gives

$$
\begin{aligned}
U(c(A)+h)-U(c(A)) & \leqslant f(A+h)-f(A) \\
& \leqslant U(c(A+h))-U(c(A+h)-h) .
\end{aligned}
$$


Dividing by $h$, letting $h \rightarrow 0$, and utilizing the continuity of $c(\cdot)$ gives

$$
f^{\prime}(A)=U^{\prime}(c(A)) \text {. }
$$

Now letting $A=y \cdot z+p(y) \cdot z$, so that $v(z, y ; p)=f(A)$, we obtain $\left(\partial v / \partial z_{i}\right)=$ $f^{\prime}(A)\left(\partial A / \partial z_{i}\right)$, as was to be shown.

With the main features of $v(z, y ; p)$ thus established, we proceed to the study of the maximum problem (i), still taking asset prices $p$ to be described by an arbitrary continuous function. The first order conditions, necessary and sufficient in this instance, are:

$$
U^{\prime}(c) p_{i}(y)=\beta \int \frac{\partial v\left(x, y^{\prime}\right)}{\partial x_{i}} d F\left(y^{\prime}, y\right) \quad(i=1, \ldots, n),
$$

provided $c, x>0$. If next period's optimum consumption $c^{\prime}$ is also positive, Proposition 2 implies in addition

$$
\frac{\partial v\left(x, y^{\prime}\right)}{\partial x_{i}}=U^{\prime}\left(c^{\prime}\right)\left[y_{i}^{\prime}+p_{i}\left(y^{\prime}\right)\right] \quad(i=1, \ldots, n) .
$$

Now in equilibrium (condition (ii)) $z=x=\underline{1}, c=\Sigma_{j} y_{j}$, and $c^{\prime}=\Sigma_{j} y_{j}^{\prime}$. Combining (3) and (5) and using these facts gives

$$
U^{\prime}\left(\sum_{j} y_{j}\right) p_{i}(y)=\beta \int U^{\prime}\left(\sum_{j} y_{j}^{\prime}\right)\left(y_{i}^{\prime}+p_{i}\left(y^{\prime}\right)\right) d F\left(y^{\prime}, y\right),
$$

for $i=1, \ldots, n$. One may think of (6), loosely, as equating the marginal rate of substitution of current for future consumption to the market rate of transformation, as given in the market rate of return on security $i$. Mathematically, (6) is a stochastic Euler equation. It is conceptually the same as equations (8) in [10].

Since equation (6) does not involve the particular value function $v(z, y ; p)$ used in its derivation, it must hold for any equilibrium price function. Conversely, if $p^{*}(y)$ solves $(6)$ and $v\left(z, y ; p^{*}\right)$ is as constructed in Proposition 1 , then the pair $\left(p^{*}(y), v\left(z, y ; p^{*}\right)\right)$ is an equilibrium. Thus solutions to (6) and equilibrium price functions are coincident.

To study (6), define

$$
g_{i}(y)=\beta \int U^{\prime}\left(\sum_{j} y_{j}^{\prime}\right) y_{i}^{\prime} d F\left(y^{\prime}, y\right) \quad(i=1, \ldots, n) .
$$

Then if the $n$ independent functional equations

$$
f(y)=g_{i}(y)+\beta \int f\left(y^{\prime}\right) d F\left(y^{\prime}, y\right)
$$$$
(i=1, \ldots, n)
$$

have solutions $\left(f_{1}(y), \ldots, f_{n}(y)\right)$, the price functions

$$
p_{i}(y)=\frac{f_{i}(y)}{U^{\prime}\left(\sum_{j} y_{j}\right)}
$$

$$
(i=1, \ldots, n)
$$


will solve (6), and $p(y)=\left(p_{1}(y), \ldots, p_{n}(y)\right)$ will be the equilibrium price function.

If $f$ is any continuous, bounded, nonnegative function on $E^{n+}$, the function $T_{i} f: E^{n+} \rightarrow R^{+}$given by

$$
\left(T_{i} f\right)(y)=g_{i}(y)+\beta \int f\left(y^{\prime}\right) d F\left(y^{\prime}, y\right)
$$

is well-defined and continuous in $y$. Since $U$ is concave and bounded (by $B$, say) we have for any $c$ :

$$
0=U(0) \leqslant U(c)+U^{\prime}(c)(-c) \leqslant B-c U^{\prime}(c)
$$

so that $c U^{\prime}(c) \leqslant B$ for all $c$. It follows that the functions $g_{i}(y)$ are bounded, since they are nonnegative and their sum is bounded by $\beta B$. Then the operators $T_{i}$ defined by (9) take elements of the space $L^{n+}$ of continuous, bounded functions into the same space. Evidently, solutions to $T_{i} f=f$ are solutions to (7), and conversely. We have, then, the following proposition.

Proposition 3: There is exactly one continuous, bounded solution $f_{i}$ to (7) (or to $\left.T_{i} f=f\right)$. For any $f_{0} \in L^{n+}, \lim _{n \rightarrow \infty} T^{n} f_{0}=f_{i}$.

The proof follows from the fact that $T_{i}$ is a contraction, verified as in the proof of Proposition 1.

In summary, we have learned that there is exactly one equilibrium price function for this economy, and we have in (6) (equivalently in (7) and (8)) an equation useful in characterizing it. In the next two sections, we develop further results at this "general" level, and then turn to the study of the nature of equilibrium prices in special cases.

\section{A "DUALITY THEOREM"}

There is a second way to construct the equilibrium price function, as will be shown in this section. Since the preceding section already provides one way, this method appears somewhat redundant in the present context. The second method is slightly more general however (since it does not require differentiability of $U$ ); it is also suggestive for stability theory.

Consider the functional equation

$$
\begin{aligned}
& r(z, y)=\inf _{q \in E^{n+}} {\left[\sup _{c, x}\left\{U(c)+\beta \int r(x, y)^{\prime} d F\left(y^{\prime}, y\right)\right\}\right] } \\
& \text { subject to } c+q \cdot x \leqslant y \cdot z+q \cdot z .
\end{aligned}
$$

It will turn out that optimal policy functions $q(z, y)$ for this dynamic program are, when evaluated at $(1, y)$, equivalent to the equilibrium price functions found in Section 4. 
To study (10), let $B$ be the space of bounded integrable functions on $E^{n+} \times$ $E^{n+}$, and let $M: B \rightarrow B$ be the operator such that (10) is equivalent to: $r=M r$. For the record, we have the following proposition.

Proposition 4: There is exactly one bounded integrable function $r$ satisfying $r=M r$, and for any $u \in B, \lim _{n \rightarrow \infty} M^{n} u=r$.

The proof parallels that of Proposition 1, and will be omitted. In fact, much more can be said about the function $r$.

Proposition 5: The solution $r$ to (10) satisfies

$$
r(z, y)=U(y \cdot z)+\beta \int r\left(z, y^{\prime}\right) d F\left(y^{\prime}, y\right) .
$$

Further, $r$ is continuous, and nondecreasing and concave in $z$ for each fixed $y$.

ProOF: Define $R: L^{2 n+} \rightarrow L^{2 n+}$ by

$$
(R w)(z, y)=U(y \cdot z)+\beta \int w\left(z, y^{\prime}\right) d F\left(y^{\prime}, y\right)
$$

so that (11) reads: $r=R r$. We show that if $w$ is continuous, and non-decreasing and concave in $z$ for each $y$, then (i) $R w$ has these properties, (ii) $M w=R w$.

The proof of (i) parallels arguments in the proof of Proposition 1, and can be omitted.

To prove (ii), observe that the point $(c, x)=(y \cdot z, z)$ satisfies $c+q \cdot x \leqslant$ $y \cdot z+q \cdot z$ for all $q$, so that $M w \geqslant R w$. Since $w$ is concave, for any $(z, y)$ the set

$$
A=\left\{(c, x): U(c)+\beta \int w\left(x, y^{\prime}\right) d F\left(y^{\prime}, y\right) \geqslant(R w)(z, y)\right\}
$$

is convex. From the separation theorem for convex sets, there is a number $a_{0}$ and a vector $a \in E^{n}$ (not both zero) such that $(c, x) \in A$ implies $a_{0} c+a \cdot x \geqslant$ $a_{0} y \cdot z+a \cdot z$. Since $U(c)$ is strictly increasing, it follows that $a_{0}>0$ and a $a \geqslant 0$, so we can define $q=\left(a / a_{0}\right)$ and write

$$
(c, x) \in A \text { implies } c+q \cdot x \geqslant y \cdot z+q \cdot z \text {. }
$$

Now for this vector $q$, suppose there is a $(c, x)$ in the interior of $A$ with $c+q \cdot x=y \cdot z+q \cdot z$. Then by reducing $c$ slightly, we obtain a point $\left(c^{\prime}, x\right)$ in $A$ such that $c^{\prime}+q \cdot x<y \cdot z+q \cdot z$ : a contradiction to (12). This proves that $q$ attains $M w$, or that $M w=R w$.

Finally, the properties listed for $r$ follow easily from the fact that $r$ solves (11), using the methods applied to the proof of Proposition 1. This completes the proof.

As immediate corollaries, we have the following propositions.

Proposition 6: For all $y, r(1, y)=v(1, y)$.

Proof: From the definition of equilibrium $v$ is the solution to (11) with $z=\underline{1}$. 
Proposition 7: If $p(y)$ is an equilibrium price function, then $q(1, y)=p(y)$ attains $r(1, y)$.

The converse to Proposition? is the following.

Proposition 8: If $q(1, y)$ attains $r(1, y)$ then $p(y)=q(1, y)$ is an equilibrium price function.

ProOF: Let $q(1, y)$ attain $r(1, y)$ and suppose that $\left(c^{0}, x^{0}\right)$ uniquely attains

$$
\max _{c, x}\left\{U(c)+\beta \int r\left(x, y^{\prime}\right) d F\left(y^{\prime}, y\right)\right\}
$$

subject to

$$
c+q(1, y) \cdot x \leqslant y \cdot \underline{1}+q(1, y) \cdot 1 .
$$

If $\left(c^{0}, x^{0}\right)=\left(\Sigma_{i} y_{i}, \underline{1}\right)$, then the assertion follows from Proposition 6 and the definition of equilibrium. If $\left(c^{0}, x^{0}\right) \neq\left(\Sigma_{i} y_{i}, \underline{1}\right)$, then a convex combination of these two points is feasible for problem (13) and yields a higher value to the objective function (since $r$ is concave in $z$ and $U$ is strictly concave) contradicting the assumption that $\left(c^{0}, x^{0}\right)$ solves problem (13).

\section{STABILITY OF EQUILIBRIUM}

The preceding sections showed that there is only one way for the economy under study to be in competitive equilibrium: when all output is consumed, all asset shares are held, and asset prices follow (6), or equivalently, solve the dynamic program (10). As always, there are innumerable ways for the economy to be out of equilibrium, so we must expect any treatment of out-of-equilibrium behavior to have considerable arbitrariness, not resolvable by economic reasoning. On the other hand, the model described above "assumes" that agents know a great deal about the structure of the economy, and perform some non-routine computations. It is in order to ask, then: will an economy with agents armed with "sensible" rules-of-thumb, revising these rules from time to time so as to claim observed rents, tend as time passes to behave as described in Sections 4 and 5 ?

To sharpen this loosely posed question somewhat, let us recognize at least three different stability questions raised by this model, and dispose of two of them at once. First, in each period an ordinary "static" market clearing occurs, in which current asset prices are set. Since stability in this sense is well understood, we need add nothing here except the assumption that it always obtains. Second, agents may be in ignorance of the distribution $F\left(y^{\prime}, y\right)$ of the exogenous production shocks, and learn its characteristics only gradually. Stability in this sense, too, is a well understood problem in Bayesian decision theory [5, Ch. 10] and need not be discussed here. Finally, consumers may be in error as to the price function, or equivalently, about the distribution of future prices conditional on 
the current state, or again equivalently, about the way they wish to evaluate their end-of-period portfolio, $x$. We focus here on this last kind of disequilibrium.

The "correct" way, given preferences, to evaluate an end-of-period portfolio $x$ is to use the equilibrium value function $v: \int v\left(x, y^{\prime}\right) d F\left(y^{\prime}, y\right)$, but agents must know this, and the economy must be in equilibrium for this valuation to be correct. Suppose instead that agents use some other function $u(z, y)$, say, where $u$ is continuous, concave, and increasing in $z$, but otherwise arbitrary, so that an end-of-period portfolio $x$ is valued at $\int u\left(x, y^{\prime}\right) d F\left(y^{\prime}, y\right)$. (To retain the conveniences of the representative consumer device, we are forced to treat all agents as being wrong in the same way.) Suppose on the basis of this arbitrary portfolio evaluation formula, asset demands are drawn and a current period market clearing asset price vector $q$ is established, at which $c=\Sigma_{i} \dot{y}_{i}$ and $x=1$. Now if prices are established in this fashion, what will be the realized utility yields experienced by agents?

The answer is given by the function $(M u)(z, y)$, where $M$ is the operator defined in association with equation (10). That this is so is the content of Proposition 5: the price $q$ which attains the right side of (10) is precisely that price which clears markets, given the portfolio valuation function $u$.

If this experience is utilized by agents, they will replace the initial valuation $u$ with the value $M u$ actually experienced, then new prices will be established, and utilities $M^{2} u$ experienced, and so on. ${ }^{5}$ Since, as shown in the preceding section, $M^{n} u \rightarrow v$, where $v$ is the equilibrium value function, prices will converge to the equilibrium price function. In short, the successive approximations used in Section 5 constitute a kind of stability theory.

It is worth emphasizing that the adjustment toward equilibrium described by these successive approximations does not presuppose that agents are familiar with the theory of Markov processes or of dynamic programming; nor need agents in equilibrium be particularly skilled at responding to survey questions about future price movements. All that is required is they have consistent preferences for consumption and asset holdings (which would seem necessary for dealing in asset markets at all) and that they revise these preferences in the direction of the consumption utility actually yielded by their asset holdings.

The point of this section, it should also be said, is not that one would use any of the successive approximations $M^{n} u$ as a description of observed behavior. (This suggestion is not even operational, since $u$ was arbitrarily chosen.) It is rather to argue that there is a theoretical reason for expecting the equilibrium to be a good approximation to behavior. Certainly one would not expect to capture the creativity which is devoted to discovering and gaining from disequilibria in actual economies in any mechanical approximation routine.

\footnotetext{
${ }^{5}$ As one of the referees for this paper emphasized, the process by which $u$ is "replaced" by $M u$, $M u$ by $M^{2} u$, and so forth, might well be quite complicated to spell out. It involves "learning" a function over time by experiencing discrete values of the function $M u$ at arguments partly selected by the household $(z)$ and partly by nature $(y)$. There are many ways to formulate learning of this sort; for our purposes here, it seems simpler just to assume that households are good at it.
} 


\section{EXAMPLES}

\subsection{Linear Utility}

The case of constant marginal utility of consumption does not exactly fit the assumptions of Section 3 (it violates boundedness) but is easily handled separately, and is a useful point of departure. In this case, equation (6) reduces to

$$
p_{i}(y)=\beta E\left(y_{i}^{\prime} \mid y\right)+\beta E\left(p_{i}\left(y^{\prime}\right) \mid y\right)
$$

which may be solved for

$$
p_{i}(y)=\sum_{s=1}^{\infty} \beta^{s} E\left(y_{i, t+s} \mid y_{i t}=y\right) .
$$

That is, the price of the $i$ th asset is the expected, discounted present value of its real dividend stream, conditioned on current information $y$.

\subsection{One Asset}

It is easy to use equation (6) (or (7)) to characterize the function $p(y)$, as can be illustrated for the case of a one-asset economy. The crucial issues are the information content of the current state $y$ (that is, the way $F\left(y^{\prime}, y\right)$ varies with $y$ ) and the degree of "risk aversion" (the curvature of $U$ ). Suppose, as a first case, that $\left\{y_{t}\right\}$ is a sequence of independent random variables: $F\left(y^{\prime}, y\right)=\phi\left(y^{\prime}\right)$. Then $g(y)$ is the constant

$$
\bar{g}=\beta \int y^{\prime} U^{\prime}\left(y^{\prime}\right) d \phi\left(y^{\prime}\right)=\beta E\left[y U^{\prime}(y)\right]
$$

and calculating $f$ from (9) as $\lim _{n \rightarrow \infty} T^{n} 0$, say, we get

$$
f(y)=\frac{\bar{g}}{1-\beta}, \quad f^{\prime}(y)=0 .
$$

Then differentiating (8) gives

$$
p^{\prime}(y)=-\frac{\beta E\left[y U^{\prime}(y)\right] U^{\prime \prime}(y)}{(1-\beta)\left[U^{\prime}(y)\right]^{2}}=p(y) \cdot \frac{-U^{\prime \prime}(y)}{U^{\prime}(y)}>0 .
$$

Rearranging,

$$
\frac{y p^{\prime}(y)}{p(y)}=-\frac{y U^{\prime \prime}(y)}{U^{\prime}(y)} .
$$

That is, the elasticity of price with respect to income is equal to the Arrow-Pratt [1] measure of relative risk aversion.

In a period of high transitory income, then, agents attempt to distribute part of the windfall over future periods, via securities purchases. This attempt is frustrated (since storage is precluded) by an increase in asset prices. 
Next, we consider autocorrelated production disturbances, under a restriction which amounts to requiring that the stochastic difference equation governing $y_{t}$ have its root between zero and one: assume that $F$ is differentiable, and that its derivatives $F_{1}$ and $F_{2}$ satisfy

$$
0<-F_{2}<F_{1} \text {. }
$$

We will repeatedly apply the following lemma.

LEMMA 1: Let $F$ satisfy (15), and let $h(y)$ have a derivative bounded between 0 and $h_{M}^{\prime}>0$. Then

$$
0 \leqslant \frac{d}{d y} \int h\left(y^{\prime}\right) d F\left(y^{\prime}, y\right) \leqslant h_{M}^{\prime}
$$

Proof: Use the change of variable $u=F\left(y^{\prime}, y\right)$, and invert to get $y^{\prime}=G(u, y)$, so that $G_{2}=\left(-F_{2}\right) / F_{1}$. Then the derivative in question is

$$
\frac{d}{d y} \int_{0}^{1} h(G(u, y)) d u=\int_{0}^{1} h^{\prime}(G) G_{2}(u, y) d u
$$

and the result follows from (15).

Now from (9), for any differentiable $f$,

$$
\frac{d}{d y}(T f)(y)=g^{\prime}(y)+\beta \frac{d}{d y} \int f\left(y^{\prime}\right) d F\left(y^{\prime}, y\right)
$$

and from the definition of $g(y)$,

$$
g^{\prime}(y)=\beta \frac{d}{d y} \int U^{\prime}\left(y^{\prime}\right) y^{\prime} d F\left(y^{\prime}, y\right) .
$$

To get any information on the slope of the solution $f(y)$ to (7), then, we must begin with bounds on the derivative of $U^{\prime}(y) y$, or on $U^{\prime \prime}(y) y+U^{\prime}(y)$. (This derivative is $U^{\prime}(y)[1-R(y)$, where $R$ is the Arrow-Pratt measure of relative risk aversion, so its magnitude has received some consideration.) For the sake of discussion, take 0 and $\bar{a}$ as lower and upper bounds on $U^{\prime \prime}(y) y+U^{\prime}(y)$. Then applying Lemma 1 to (18),

$$
0 \leqslant g^{\prime}(y) \leqslant \beta \bar{a} \text {. }
$$

Then repeated application of (17), using Lemma 1 at each step, yields

$$
0 \leqslant f^{\prime}(y) \leqslant \frac{\beta \bar{a}}{1-\beta}
$$

where $f(y)$ is the solution to (8) in this one asset case. ${ }^{6}$ From (8), the elasticity of

\footnotetext{
${ }^{6}$ Differentiability of the approximations $T^{n} F$ does not imply the differentiability of $f$, and in fact, there is no easy way to verify this. For " $f^{\prime}(y) \leqslant c$ " read: " $f\left(y_{1}\right)-f\left(y_{0}\right) \leqslant c\left(y_{1}-y_{0}\right)$."
} 
the equilibrium price function is

$$
\frac{y p^{\prime}(y)}{p(y)}=\frac{y f^{\prime}(y)}{f(y)}-\frac{y U^{\prime \prime}(y)}{U^{\prime}(y)}
$$

The second term on the right of $(20)$ is the "income effect" we have seen above; it is positive. The first term might be called the "information effect"; 7 it has the sign of $f^{\prime}(y)$. Evidently, the use one can make of these formulas depends on our knowledge of the curvature of $U ;(19)$ and (20) show how to translate such knowledge into knowledge about asset prices.

In the present case of relative risk aversion ${ }^{8}$ less than unity, we have found in (19) that $f^{\prime}(y)>0$, so that the information effect is positive. Thus as one might expect, new optimistic information on future dividends leads to increased asset prices. (Of course, one might also expect that this information will lead to an attempted consumption binge now, lowering asset prices!)

Observationally, the derivative $p^{\prime}(y)$ is the change in the ratio of a comprehensive stock price index to the CPI, as real output varies. Even in the simplified economy under study, then, the relationship of asset prices to real output is far from simple and possibly not even monotonic. Perhaps it has been good judgment, not merely timidity, which has led aggregate theorists to steer clear of any attempt to "understand the market."

\subsection{Many, Independent Assets}

If the number of productive units is large, and if there is sufficient independence across units, one wo!ld expect that replacing the term $U^{\prime}\left(\Sigma_{j} y_{j}\right)$ in (6) with $U^{\prime}(\mu)$, where

$$
\mu=\sum_{j} \mu_{j}=\sum_{j} \int_{j} \phi(y) d y
$$

in mean total output, would yield a good approximation to the equilibrium price function. Let us pursue this idea, and the question of approximation generally, with the aid of the next lemma.

LEMMA 2: Let $S, T: L \rightarrow L$ be contractions with modulus $\beta$ and fixed points $f_{s}$, $f_{T} \in L$. Suppose that

$$
\|S f-T f\| \leqslant A \text { for all } f \in L \text {. }
$$

Then

$$
\left\|f_{S}-f_{T}\right\| \leqslant \frac{A}{1-\beta} .
$$

\footnotetext{
${ }^{7}$ This follows Grossman [8].

${ }^{8}$ In this multiperiod context, the term "risk aversion" is perhaps misleading, since the curvature of $U$ also governs the intertemporal substitutability of consumption. With time-additive utility, there is no way to disentangle these conceptually distinct aspects of preferences.
} 
Proof: For any $f$,

and, in general,

$$
\begin{aligned}
\left\|S^{2} f-T^{2} f\right\| & \leqslant\left\|S^{2} f-T S f\right\|+\left\|T S f-T^{2} f\right\| \\
& \leqslant\|S(S f)-T(S f)\|+\beta\|S f-T f\| \\
& \leqslant A+\beta A,
\end{aligned}
$$

$$
\left\|S^{n} f-T^{n} f\right\| \leqslant A\left(1+\beta+\ldots+\beta^{n-1}\right) .
$$

Letting $n \rightarrow \infty$ gives the result.

Now if $\tilde{g}_{i}(y)$ is an approximation to $g_{i}(y)$, and $\tilde{T}_{i}$ is defined by

we have

$$
\tilde{T}_{i} f=\tilde{g}_{i}(y)+\beta \int f\left(y^{\prime}\right) d F\left(y^{\prime}, y\right),
$$

$$
\left\|\tilde{T}_{i} f-T_{i} f\right\|=\left\|\tilde{g}_{i}(y)-g_{i}(y)\right\| .
$$

Then if $f_{i}$ and $\tilde{f}_{i}$ are the fixed points of $T_{i}$ and $\tilde{T}_{i}$, respectively, Lemma 2 gives the bound

$$
\left\|\tilde{f}_{i}-f_{i}\right\| \leqslant(1-\beta)^{-1}\left\|\tilde{g}_{i}-g_{i}\right\| .
$$

Returning to the specific approximation proposed above, let

$$
\tilde{g}_{i}(y)=U^{\prime}(\mu) \int y_{i} d F\left(y^{\prime}, y\right),
$$

define $\tilde{T}_{i}$ as above, and let $\tilde{f}_{i}$ be the fixed point of $\tilde{T}_{i}$. Then the approximate price function

$$
\tilde{p}_{i}(y)=\frac{\tilde{f}_{i}(y)}{U^{\prime}(\mu)}
$$

is just the solution calculated in 7.1 above.

To evaluate this approximation, we need bounds on $\left\|\tilde{g}_{i}-g_{i}\right\|$. To this end, let us bound $U^{\prime \prime}(y):\left\|U^{\prime \prime}\right\| \leqslant M$. Then

$$
\begin{aligned}
\left\|g_{i}(y)-\tilde{g}_{i}(y)\right\| & =\beta\left\|\int\left[U^{\prime}\left(\sum_{j} y_{j}^{\prime}\right)-U^{\prime}(\mu)\right] y_{i}^{\prime} d F\left(y^{\prime}, y\right)\right\| . \\
& \leqslant \beta M\left\|\int\left(\sum_{j} y_{j}^{\prime}-\mu\right) y_{i}^{\prime} d F\left(y^{\prime}, y\right)\right\|
\end{aligned}
$$

using the mean value theorem. If the $y_{i}$ 's are independent, or if $F\left(y^{\prime}, y\right)=$ $\Pi_{k} F_{k}\left(y_{k}^{\prime}, y_{k}\right)$, then

$$
\begin{aligned}
\int\left(\sum_{j} y_{j}^{\prime}-\mu\right) y_{i}^{\prime} d F\left(y^{\prime}, y\right)= & \int\left(\sum_{i}\left(y_{j}^{\prime}-\mu_{j}\right)\right)\left(y_{i}^{\prime}-\mu_{i}\right) d F\left(y^{\prime}, y\right) \\
& +\mu_{i} \int \sum_{j}\left(y_{j}^{\prime}-\mu_{j}\right) d F\left(y^{\prime}, y\right) \\
= & \operatorname{var}\left(y_{i}^{\prime} \mid y_{i}\right)+\mu_{i} \sum_{j} E\left(y_{j}^{\prime}-\mu_{j} y_{j}\right) .
\end{aligned}
$$


Combining gives

$$
\left.\| g_{i}(y)-\tilde{g}_{i}\right) \| \leqslant \sup _{y}\left[\operatorname{var}\left(y_{i}^{\prime} \mid y_{i}\right)+\mu_{i} \sum_{j} E\left(y_{j}^{\prime}-\mu_{j} \mid y_{j}\right)\right] .
$$

If we think of a sequence of economies of the same total size, but with more and more independent productive units of roughly equal size, $\operatorname{var}\left(y_{i}^{\prime} \mid y_{i}\right)$ and $\mu_{i} \Sigma_{j} E\left(y_{j}^{\prime}-\mu_{j} \mid y_{j}\right)$ will tend to zero, and the approximations $\tilde{f}_{i}$ will become close.

\section{THE MARTINGALE PROPERTY}

We have shown that equation (6) exhausts the implications of the assumption that, in this model economy, prices are in equilibrium and "reflect all available information." Evidently, asset prices themselves do not possess the Martingale property. The series that does have this property (something has to, in this time-additive set up) is the series $w_{i t}(i=1, \ldots, n)$ defined by

$$
w_{i, t+1}-w_{i t}=\beta U^{\prime}\left(\sum_{j} y_{j, t+1}\right)\left(y_{i, t+1}+p_{i, t+1}\right)-U^{\prime}\left(\sum_{j} y_{j t}\right) p_{i t}
$$

since from (6), the expectation of the right side of (22), conditioned on all available information (in this case, $y_{t}$ ) is zero.

If the terms $U^{\prime}\left(\Sigma_{j} y_{j t}\right)$ do not vary much, either because agents are indifferent to risk (example 7.1) or because there is little aggregate risk (example 7.3), then securities prices properly "corrected" for dividends $y_{i t}$ almost have the property but not without another "correction" for the discount factor $\beta$. In any case, neither rationale for a constant $U^{\prime}\left(\Sigma_{j} y_{j t}\right)$ seems likely to closely approximate reality.

It should be added that the importance of the requirement that "the conditions of market equilibrium can be stated in terms of expected returns" has been repeatedly emphasized by Fama and other efficient market theorists; it is not a new result from this paper. What is new, I think, is an explicit framework within which one can judge what this requirement means and whether or not it is satisfied, or which in other words can lend some insight into the conditions under which the Martingale property is likely to approximately describe a price series. Within this framework, it is clear that the presence of a diminishing marginal rate of substitution of future for current consumption is inconsistent with this property. ${ }^{9}$

\section{CONCLUSIONS}

What can be concluded from this exercise (beyond the observation that a little knowledge of geometric series goes a long way, or perhaps, is a dangerous thing)? Substantively, the discussion of stability of Section 6 indicates that the

\footnotetext{
${ }^{9}$ This complements Danthine's [4] finding that a diminishing marginal rate of transformation over time, in a model with storage, has the same effect.
} 
applicability of the hypothesis that agents "know" the "true" probability distributions of future prices has little to do with the question of whether agents (ourselves included) think of, or describe, their behavior in these terms. A relatively crude use of hindsight, applied in a reasonably stationary physical environment, will lead to behavior well-approximated by rational expectations.

With respect to the random character of stock prices, it is evident that one can construct rigorous economic models in which price series have this characteristic ${ }^{10}$ and ones with equally rational and well-informed agents in which they do not. This would suggest that the outcomes of tests as to whether actual price series have the Martingale property do not in themselves shed light on the generally posed issue of market "efficiency."

In the main, however, this paper is primarily methodological: an illustration of the use of some methods which may help to bring financial and economic theories closer together. It may help, then, to close with some guesses as to the fronts on which further progress can be expected.

The time-additive preference structure is, as remarked earlier, a nuisance, and it has no rationale beyond tractability. It would not be difficult (with the aid of [6]) to use recursive, but non-additive preferences of the Koopmans-DiamondWilliamson [9] type, provided sufficient "impatience" is assumed.

Second, one would like to introduce capital accumulation. In this regard, the marginal analysis of Section 4 is probably a dead-end: equation (6) is a kind of Euler condition, and will necessarily involve capital provided capital enters the model in a non-trviial way. Aside from special cases (such as the one studied in Section 4) stochastic Euler equations are not likely to be of value in constructing solutions. Equation (10) in Section 5 appears more promising; perhaps it has useful analogues in more generally formulated models.

\section{University of Chicago}

Manuscript received September, 1975; final revision received March, 1978.

\section{REFERENCES}

[1] Arrow, Kenneth J.: Aspects of the Theory of Risk Bearing. Helsinki: Yrjo Jahnsson Lectures, 1965.

[2] Berge, Claude: Topological Spaces. New York: Macmillan, 1963.

[3] BlaCkWell, David: "Discounted Dynamic Programming," Annals of Mathematical Statistics, 36 (1965), 226-235.

[4] Danthine, Jean-Pierre: "On the Relevance of the Efficient Market Model," CarnegieMellon University Working Paper, 1974.

[5] De Groot, Morris H.: Optical Statistical Decisions. New York: McGraw-Hill, 1969.

[6] Denardo, ERIC V.: "Contraction Mappings in the Theory Underlying Dynamic Programming," SIAM Review, 9 (1967), 165-177.

[7] FAMA, Eugene F.: "Efficient Capital Markets: A Review of Theory and Empirical Work," Journal of Finance, 25 (1970), 387-417.

[8] Grossman, SANDFORD: "The Existence of Futures Markets, Noisy Expectations, and Informational Externalities," unpublished University of Chicago Working Paper, 1975.

${ }^{10}$ Samuelson did this in [14]. 
[9] Koopmans, Tualling C., Peter A. Diamond, and Richard E. Williamson: "Stationary Utility and Time Perspective," Econometrica, 32 (1964), 82-100.

[10] LeRoy, STEPHEN F.: "Risk Aversion and the Martingale Property of Stock Prices," International Economic Review, 14 (1973), 436-446.

[11] — : "The Determination of Stock Prices," unpublished University of Pennsylvania Doctoral Dissertation, 1971.

[12] LuCAs, Robert E., JR., ANd Edward C. Prescott: "Investment under Uncertainty," Econometrica, 39 (1971), 659-681.

[13] Muth, JoHn F.: "Rational Expectations and the Theory of Price Movements," Econometrica, 29 (1961), 1-23.

[14] Samuelson, Paul A.: "Proof that Properly Anticipated Prices Fluctuate Randomly," Industrial Management Review, 6 (1965), 41-49. 